\title{
Correction to: Diffusion-weighted MRI improves response assessment after definitive radiotherapy in patients with NSCLC
}

Philippe Jagoda ${ }^{1 *}$, Jochen Fleckenstein ${ }^{2}$, Mathias Sonnhoff ${ }^{2}$, Günther Schneider ${ }^{1}$, Christian Ruebe ${ }^{2}$, Arno Buecker ${ }^{1}$ and Jonas Stroeder ${ }^{1}$

Correction to: Cancer Imaging 21, 15 (2021)

https://doi.org/10.1186/s40644-021-00384-9

After publication of the original article [1], the authors identified that authors' names have been wrongly marked. The given name and family name were erroneously transposed.

The incorrect authors' names are:

$<$ GivenName> Jagoda </GivenName > < FamilyName> Philippe $<$ /FamilyName $>$

$<$ GivenName $>$ Fleckenstein </GivenName $>$ < FamilyName $>$ Jochen $</$ FamilyName $>$

$<$ GivenName $>$ Sonnhoff $</$ GivenName $>$ <amilyName $>$ Mathias $<$ /FamilyName $>$

$<$ GivenName $>$ Schneider </GivenName $>$ < FamilyName $>$ Günther $</$ FamilyName $>$

<GivenName> Ruebe </GivenName> < FamilyName> Christian $<$ /FamilyName $>$

$<$ GivenName $>$ Buecker </GivenName $>$ < Family-

Name $>$ Arno $<$ /FamilyName $>$

$<$ GivenName $>$ Stroeder </GivenName $>$ < Family-

Name $>$ Jonas </FamilyName $>$

The correct authors' names are:

$<$ GivenName $>$ Philippe </GivenName $>$ < FamilyName $>$ Jagoda $<$ /FamilyName $>$

The original article can be found online at https://doi.org/10.1186/s40644021-00384-9.

* Correspondence: philippe.jagoda@uks.eu

${ }^{1}$ Clinic for Diagnostic and Interventional Radiology, Saarland University

Medical Center, Kirrberger Str. 1, 66421 Homburg, Saar, Germany

Full list of author information is available at the end of the article
<GivenName > Jochen </GivenName > < FamilyName > Fleckenstein $<$ /FamilyName $>$

$<$ GivenName $>$ Mathias </GivenName > FamilyName $>$ Sonnhoff $<$ /FamilyName $>$

<GivenName> Günther </GivenName> < FamilyName $>$ Schneider $</$ FamilyName $>$

$<$ GivenName $>$ Christian </GivenName $>$ < FamilyName $>$ Ruebe $<$ /FamilyName $>$

$<$ GivenName $>$ Arno </GivenName $>$ < FamilyName $>-$ Bueker $<$ /FamilyName $>$

$<$ GivenName $>$ Jonas $</$ GivenName $><$ FamilyName $>$ Stroeder $<$ /FamilyName $>$

The original article has been corrected.

\section{Author details \\ ${ }^{1}$ Clinic for Diagnostic and Interventional Radiology, Saarland University \\ Medical Center, Kirrberger Str. 1, 66421 Homburg, Saar, Germany. \\ ²Department of Radiotherapy and Radiation Oncology, Saarland University \\ Medical Center, Kirrberger Str. Geb. 6.5, 66421 Homburg, Saar, Germany.}

Published online: 09 February 2021

Reference

1. Jagoda, et al. Diffusion-weighted MRI improves response assessment after definitive radiotherapy in patients with NSCLC. Cancer Imaging. 2021;21:15. https://doi.org/10.1186/s40644-021-00384-9.

(c) The Author(s). 2021 Open Access This article is licensed under a Creative Commons Attribution 4.0 International License, which permits use, sharing, adaptation, distribution and reproduction in any medium or format, as long as you give appropriate credit to the original author(s) and the source, provide a link to the Creative Commons licence, and indicate if changes were made. The images or other third party material in this article are included in the article's Creative Commons licence, unless indicated otherwise in a credit line to the material. If material is not included in the article's Creative Commons licence and your intended use is not permitted by statutory regulation or exceeds the permitted use, you will need to obtain permission directly from the copyright holder. To view a copy of this licence, visit http://creativecommons.org/licenses/by/4.0/ The Creative Commons Public Domain Dedication waiver (http://creativecommons.org/publicdomain/zero/1.0/) applies to the data made available in this article, unless otherwise stated in a credit line to the data. 